\title{
Application of frequency-domain algorithms in ultrasound imaging of composite materials
}

\author{
Dmitry Dolmatov ${ }^{1, a}$ and Vladislava Abramets ${ }^{1}$ \\ ${ }^{1}$ National Research Tomsk Polytechnic University, 634050 Tomsk, Russia
}

\begin{abstract}
Non-destructive ultrasonic testing of composite materials requires imaging algorithms which could be applied for anisotropic structures. At present, time-domain techniques are used for this purpose. However, it is evident that this method has lower computational speed compared with frequency-domain algorithms. This paper discusses advanced approach off frequency-domain techniques for multilayer composite materials inspection. The proposed method is based on multi-layer omega-k (MULOK) ultrasound frequency-domain imaging algorithms. It is shown that MULOK algorithms enable highly accurate reconstruction within a higher computational speed.
\end{abstract}

\section{Introduction}

Composite materials are used in aerospace engineering extensively. Application of these structures requires nondestructive testing that enables to reduce the risk of accidents. One of the methods, which could be used for this purpose, is ultrasonic nondestructive testing [1]. Furthermore, advanced ultrasonic imaging algorithms allow accurate determination of defect and its size, shape and location in the controlled object. That information is to be used for reliability assessment of composite structures. However, ultrasonic visualization and image reconstruction of anisotropic structure of composite materials is one of the major challenges due to the acoustic properties changes within the direction of the wave.

\section{Ultrasound imaging algorithms}

Synthetic aperture focusing technique (SAFT) used to be the first ultrasound imaging algorithm [2]. This algorithm proposes image reconstruction according to the following formula:

$$
I(x, z)=\left|\sum_{t r=1}^{N} P\left(\tau, x_{t r}\right)\right|
$$

$P\left(\tau, x_{t r}\right)$ is a value of ultrasound field in the position of ultrasound transducer $x_{t r}$ at the time $\tau$. Correlation between $\tau$ and coordinates of the image $x$ and $z$ is following:

\footnotetext{
${ }^{\mathrm{a}}$ Corresponding author : dolmatovdo@tpu.ru
} 


$$
\tau=\frac{2 \sqrt{\left(x-x_{t r}\right)^{2}+z^{2}}}{C_{l}}
$$

$C_{l}$ is the speed of longitudinal ultrasonic waves in the specimen. This algorithm can be applied only in the case of isotropic materials. Further, SAFT was adjusted for multilayer structures imaging [3] that could be used in ultrasonic imaging of composite materials. However, it is known that frequency-domain algorithms have better computational speed than time-domain algorithms [4]. Frequency-domain algorithms can be implemented in two ways. First algorithm was proposed by Gazdag [5] and was used in seismology. This technique is known as phase shift migration (PSM) algorithm. Afterwards, it was improved and successfully applied for ultrasonic nondestructive testing [6]. The imaging algorithm implies slice by slice reconstruction of the image according to the following correlation:

$$
I(x, z+\Delta z)=\int_{-\infty}^{+\infty}\left(\sum_{\omega} P\left(\omega, k_{x}, z+\Delta \mathrm{z}\right)\right) \cdot e^{i k_{x} x}
$$

Each slice of the image is a result of inverse Fourier transformation of $\operatorname{sum} P\left(\omega, \mathrm{k}_{x}, z+\Delta \mathrm{z}\right)$ along the wave number $\mathrm{k}_{x}$. Function $P\left(\omega, \mathrm{k}_{x}, z+\Delta \mathrm{z}\right)$ is the extrapolated wave field, which is derived into two steps. First, 2-D Fourier transformation of registered wave field is conducted:

$$
P\left(\omega, k_{x}, z\right)=\frac{1}{4 \pi^{2}} \int_{-\infty}^{+\infty} \int_{-\infty}^{+\infty} P(t, x, z) \cdot e^{-i\left(k_{x} x-\omega t\right)} d x d t
$$

Then, the wave field is extrapolated to the depth $z+\Delta z$ :

$$
P\left(\omega, k_{x}, z+\Delta z\right)=P\left(\omega, k_{x}, z\right) \cdot e^{\left(i \cdot k_{z}\left(\omega, k_{x}\right) \cdot \Delta z\right)}
$$

Correlation between wave numbers $k_{z}, k_{x}$ and temporal frequency $\omega$ is following:

$$
k_{z}\left(\omega, k_{x}\right)=\sqrt{\frac{4 \omega^{2}}{C_{l}^{2}}-k_{x}^{2}}
$$

Stolt [7] proposed second approach. This method suggests image reconstruction according to the following correlation:

$$
I(x, z)=\iint\left(\frac{C_{l}}{2} \frac{k_{z}}{\sqrt{k_{x}^{2}+k_{z}^{2}}}\right) P\left(k_{x}, k_{z}\right) e^{-i k_{x} x-i k_{z} z} d k_{x} d k_{z}
$$

The main part of this algorithm is the change of the variable. Temporal frequency $w$ is changed to the wave number $k_{z}$ :

$$
w\left(k_{x}, k_{z}\right)=\frac{C_{l}}{2} \cdot \sqrt{k_{x}^{2}+k_{z}}
$$

This algorithm can be applied only for isotropic materials. However, it can be adapted for multilayer structures testing by combination this algorithm with PSM technique [8]. Initially, first layer is reconstructed by using (7). After that, wave field is extrapolated according to (5) to the depth where layer with new properties starts. The last step is wave field extrapolation that is used in Stolt 
algorithm for reconstruction of second layer and so forth. Application of this approach in ultrasound imaging is known as Multilayer omega-k (MULOK) algorithm [4].

\section{Experimental procedure}

A number of simulated processing was performed in Matlab R2009b (The MathWorks, Natick, MA) in order to compare two algorithms. The simulations were made on a dual-core $2 \mathrm{MHz}, 8 \mathrm{~Gb} \mathrm{RAM}$ computer. Simulated material consists of two layers. First layer was made of copper, second layer was made of aluminum. The thickness of both layers was $25 \mathrm{~mm}$. The length of simulated specimen was $40 \mathrm{~mm}$. The simulated specimen is shown in the Figure 1.

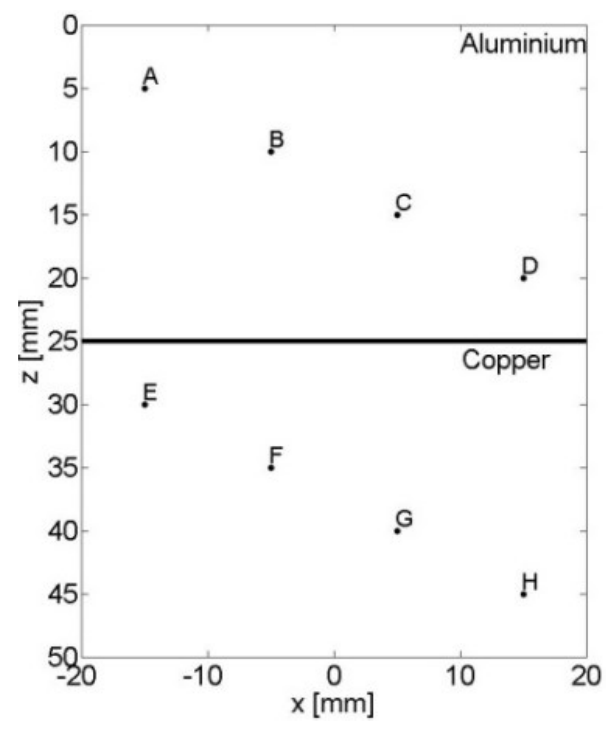

Figure 1.The simulated specimen.

The transducer which was used in this simulation had $5 \mathrm{MHz}$ frequency and diameter of $3 \mathrm{~mm}$. Sampling frequency was $50 \mathrm{MHz}$. The step of the transducer was changed for each simulation. Four simulations were made for each algorithm. The step values were $1,0.5,0.2$ and 0.1 millimeters. The results of simulations are shown in the Figure 2 for PSM and in the Figure 3 for MULOK.

The results of the simulation were analyzed in order to estimate the computational speed and the quality of the reconstructed image. First parameter can be defined by measuring elapsed time for each simulation. Correlation between calculation time and number of steps in each simulation is presented in the Figure 4. Quality of the reconstructed image can be evaluated according to the following relation [9]:

$$
P=\frac{A^{-6 d B}}{\lambda_{c}^{2}}
$$

$A$ is an area where a point spread function is greater than $-6 \mathrm{~dB}$ down from its local maximum value whereas $\lambda_{c}$ is a transducer center wavelength. This value was determined for both algorithms at the step $0.1 \mathrm{~mm}$. The results are presented in the Table 1 . The difference between values for both algorithms was derived by using the following correlation:

$$
D=\frac{P^{P S M}-P^{M U L O K}}{P^{P S M}} \cdot 100 \%
$$


MATEC Web of Conferences

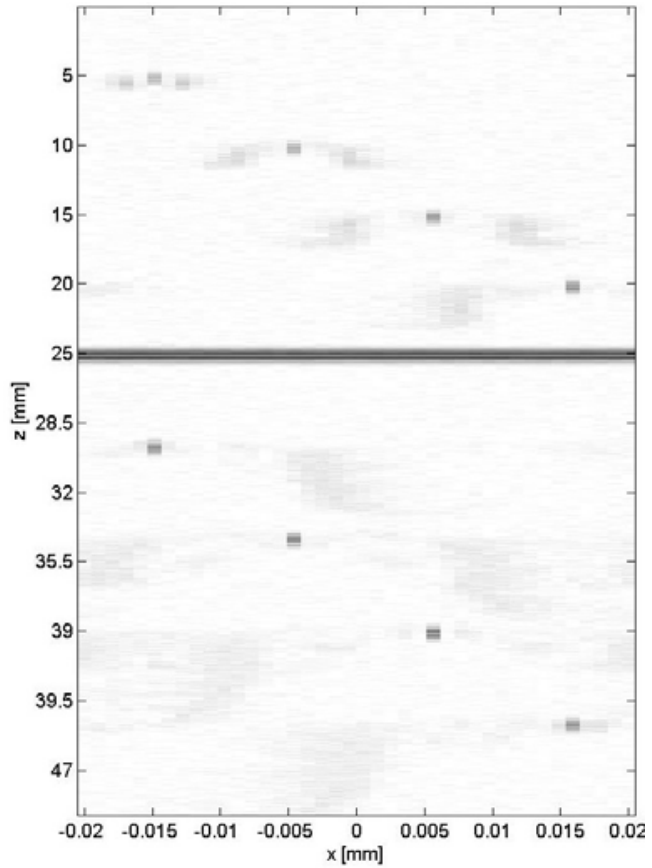

(a)

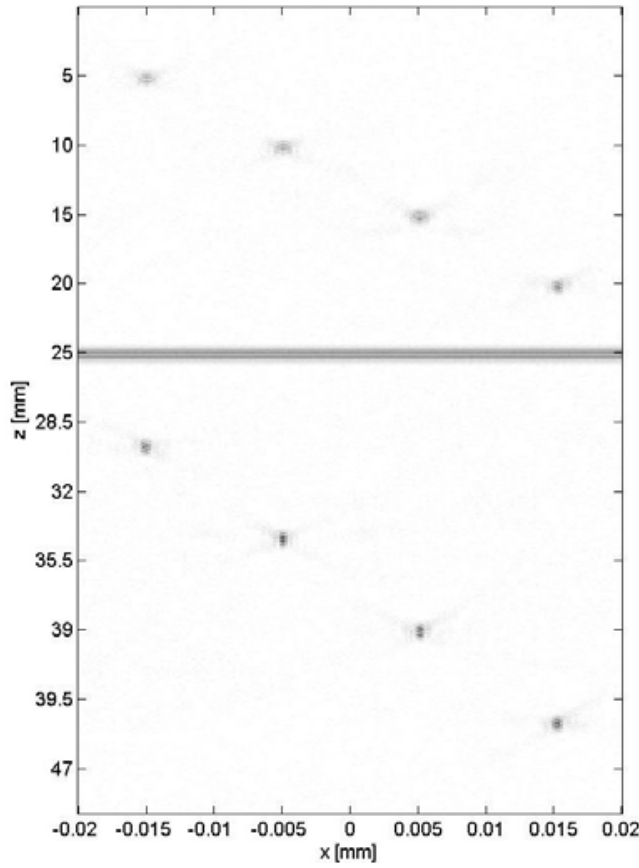

(c)

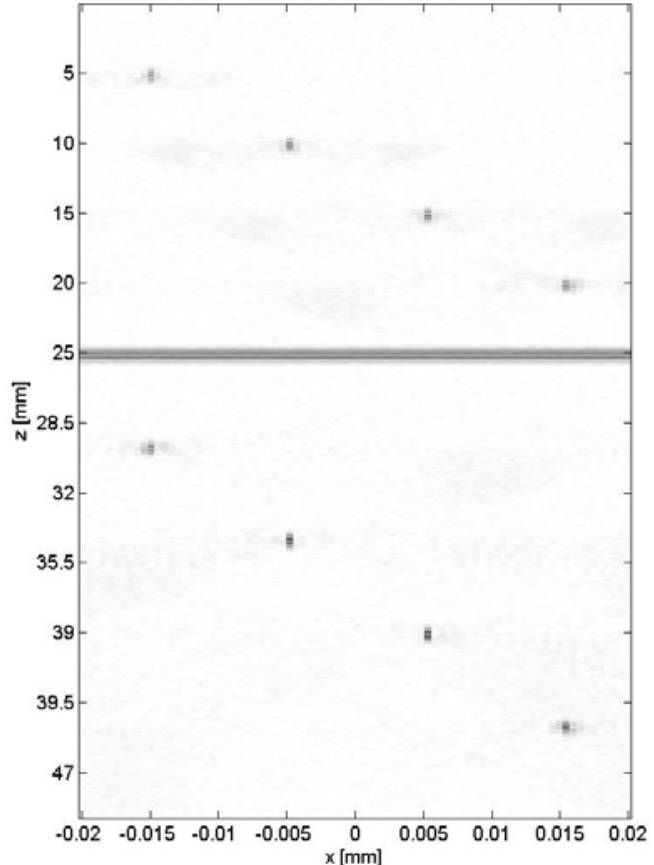

(b)

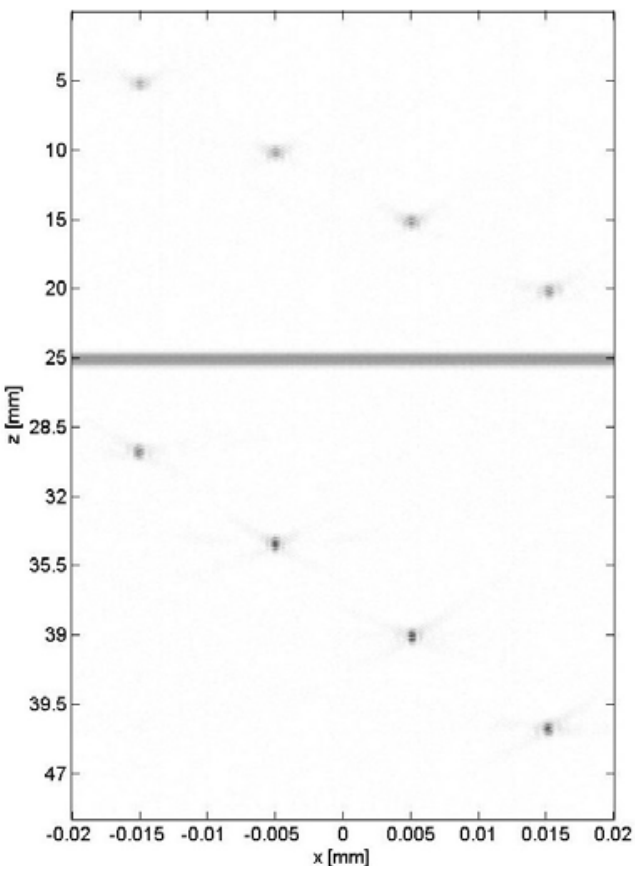

(d)

Figure 2.The results of simulations by using PSM algorithm: (a) $1 \mathrm{~mm}$ transducer step, (b) $0.5 \mathrm{~mm}$ transducer step, (c) $0.2 \mathrm{~mm}$ transducer step, (d) $0.1 \mathrm{~mm}$ transducer step. 
Space Engineering 2016

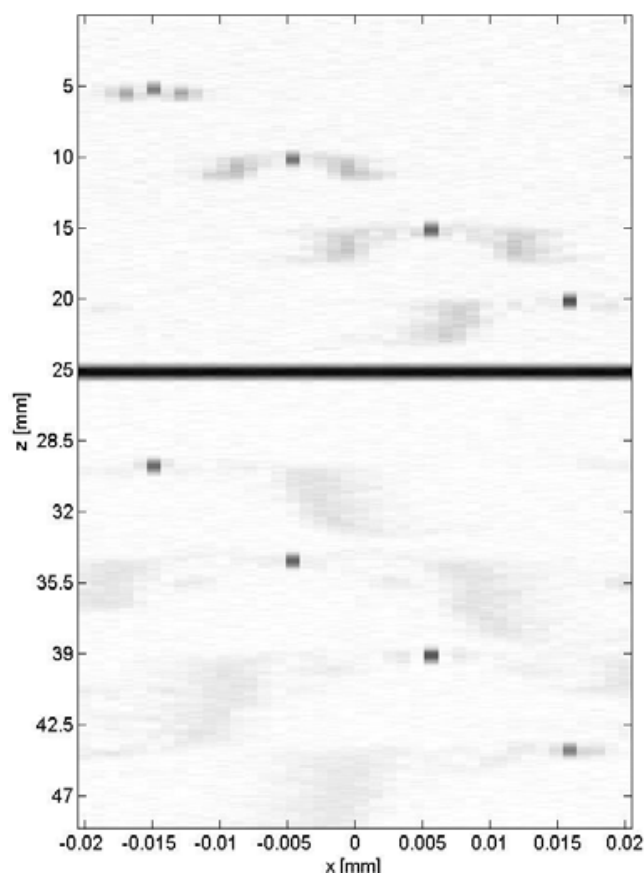

(a)

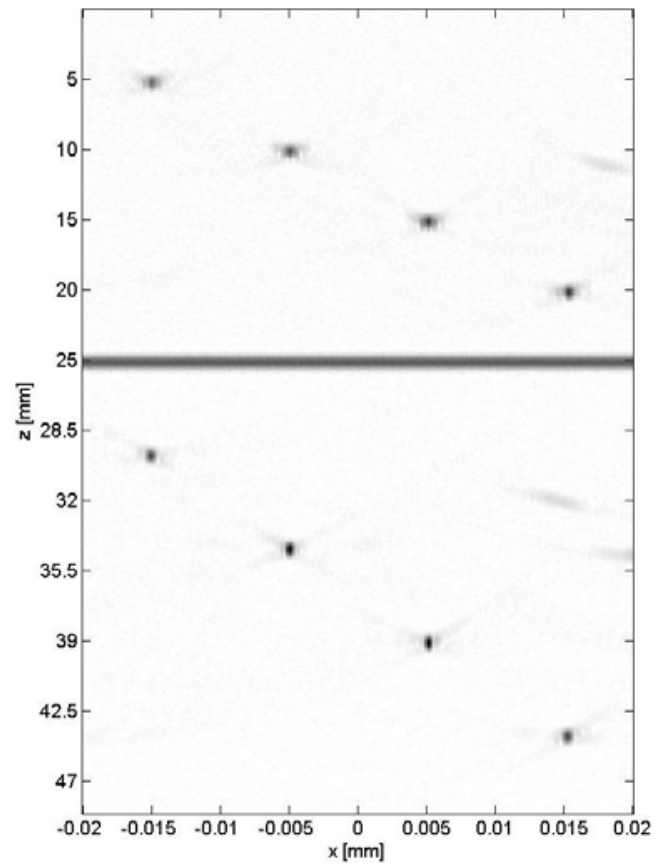

(c)

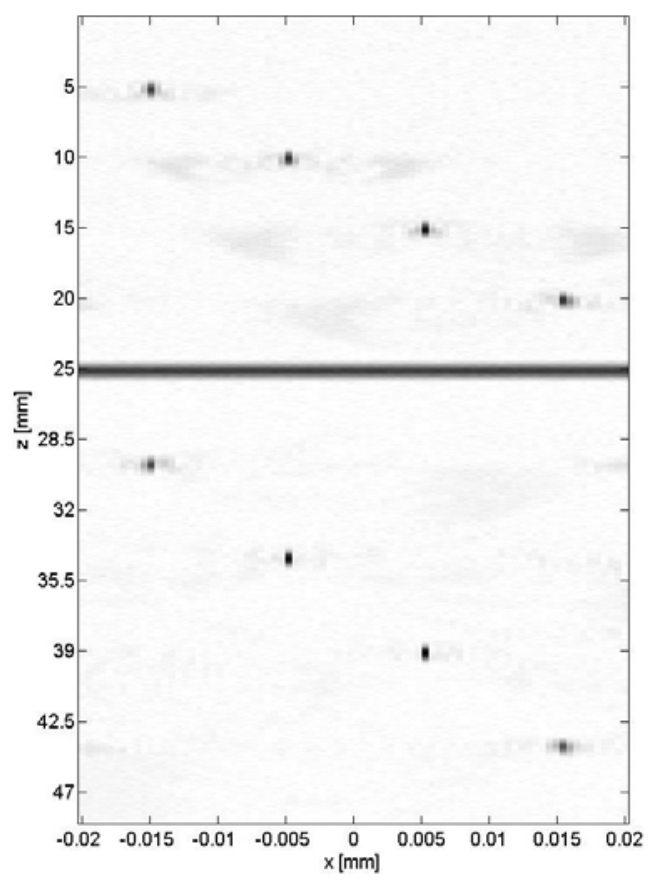

(b)

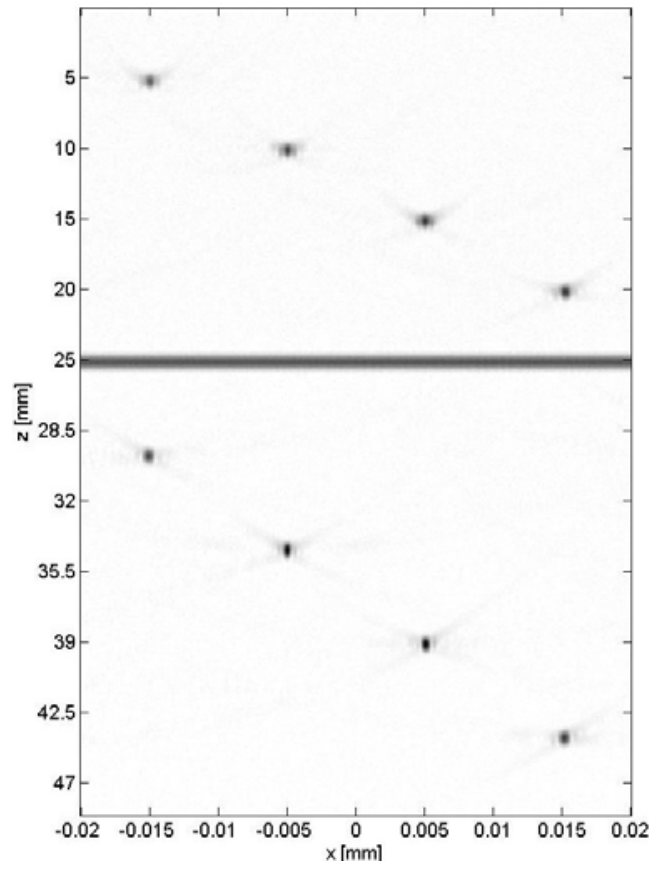

(d)

Figure 3. The results of simulations by using MULOK algorithm: (a) $1 \mathrm{~mm}$ transducer step, (b) $0.5 \mathrm{~mm}$ transducer step, (c) $0.2 \mathrm{~mm}$ transducer step, (d) $0.1 \mathrm{~mm}$ transducer step. 
MATEC Web of Conferences

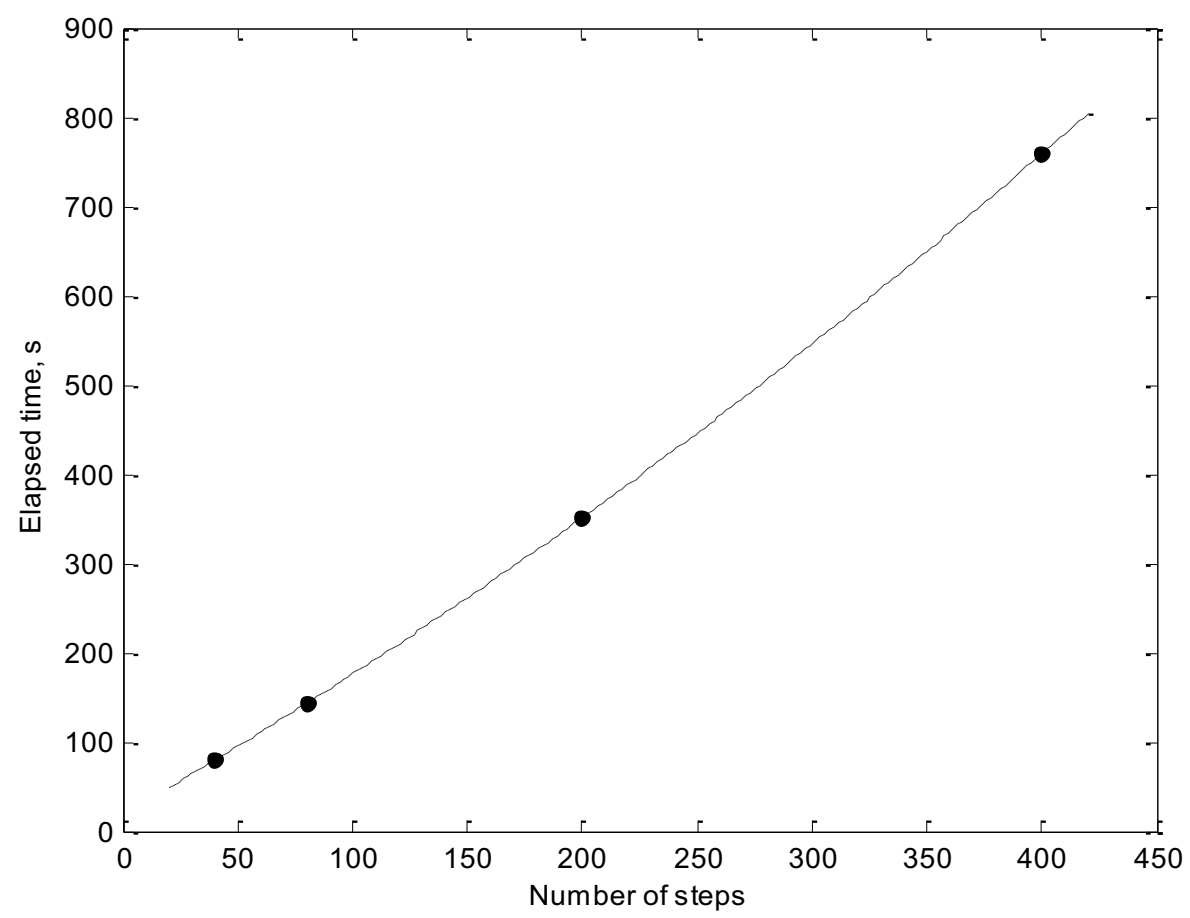

(a)

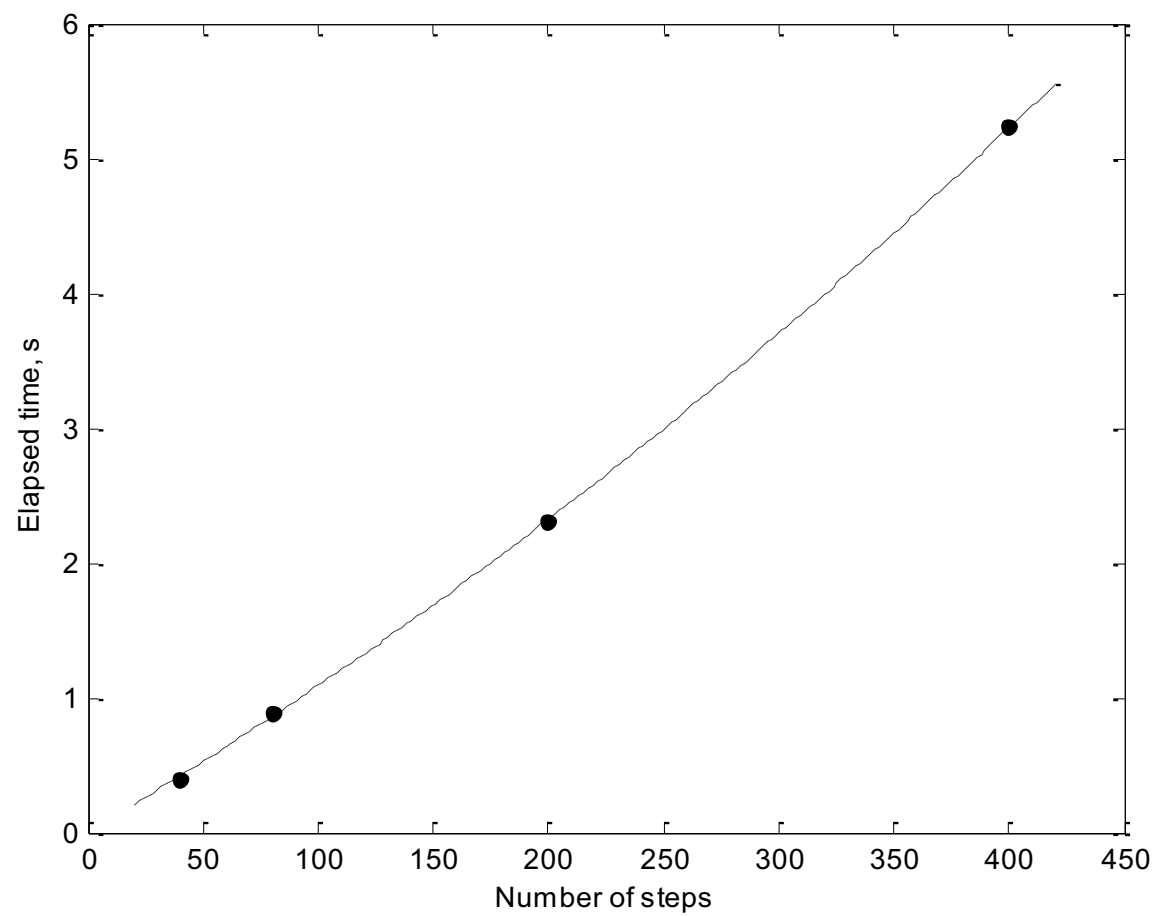

(b)

Figure 4. The correlation between calculation time and number of steps in each simulation: (a) PSM, (b) MULOK. 
Table 1. Performance indicators of MULOK and PSM algorithms.

\begin{tabular}{|c|c|c|c|}
\hline Point & MULOK & PSM & Difference, $\%$ \\
\hline A & 2,13 & 2,18 & 2.1 \\
\hline B & 2,17 & 2,18 & 0.5 \\
\hline C & 2,22 & 2,22 & 0 \\
\hline D & 1,78 & 1,91 & 7.6 \\
\hline E & 1,91 & 1,91 & 2.9 \\
\hline F & 1,40 & 1,49 & 6.1 \\
\hline G & 1,46 & 1,51 & 3.9 \\
\hline H & 1,89 & 1,90 & 0.7 \\
\hline
\end{tabular}

Comparison of the obtained results demonstrates that MULOK algorithm is faster than PSM. Computational time of imaging algorithm is very important in ultrasound imaging due to the fact that in nondestructive testing it is necessary to work with huge set of data. Also, Table 1 represents that the quality of the images for both methods is almost the same. That is why, experimental results reveal that MULOK algorithm enables effective ultrasound imaging for multilayer materials within a higher computation speed.

\section{Conclusion}

This study represents that frequency-domain ultrasound imaging algorithm can be used for anisotropic materials, which consist of isotropic layers. There were two-dimensional case and two layers case studied separately. However, the same simulation can be made for three-dimensional case and multilayer material if it is required. The obtained results demonstrated that MULOK algorithm can be successfully applied in practice. However, this algorithm is not to be used for ultrasound imaging of all types of composite materials due to the diversity of its structure and properties. It is possible to apply MULOK algorithm for composite materials which are meet the conditions of simulations.

\section{Acknowledgement}

Research has been conducted with financial support of the Federal Target Program, project unique identifier is RFMEFI57514X0048.

\section{References}

1. G. Scott, C. M. Scala, NDTi 15.2, 75 (1982)

2. S. R. Doctor, T. E. Hall, L. D. Reid, NDTi 19.3, 163 (1986)

3. M. H. Skjelvareid, Y. Birkelund, ASME 2010 Pressure Vessels and Piping Division/K-PVP Conference (2010)

4. M. H. Skjelvareid, UFFC 58.5, 1037 (2011)

5. J. Gazdag, G. 43.7, 1342 (1978)

6. T. Olofsson, UFFC 57.11, $2522(2010)$

7. R. H. Stolt, G. 43.1, 23 (1978)

8. Y. C. Kim, R. Gonzalez, J. R. Berryhill, G. 54.3, 319 (1989)

9. A. J. Hunter, B. W. Drinkwater, P. D. Wilcox, UFC 55.11, 2450 (2008)

10. K. G. Kvasnikov, A. I. Soldatov , I. O. Bolotina, M. K. Krening, A. A. Potapenko, RJNT 49.11, 625 (2013)

11. D. Sednev, A. Lider, D. Demyanuk, M. Kroening, Y. Salchak, PP 70, 505 (2015) 